\title{
Correspondence
}

Psychological Medicine, 48 (2018).

doi:10.1017/S0033291717002574

First published online 14 September 2017

\section{Authors' reply: Psychosis Spectrum Disorder is a clinical diagnosis}

We appreciate both Curtis and Derks, and Bora for their interest in our recent viewpoint on the concept of psychosis spectrum disorder (PSD) (Guloksuz \& van Os, 2017). Both letters in response to our paper raise several concerns about the PSD framework, in particular extending the boundaries of PSD to include psychotic experiences. We welcome the opportunity to reiterate our understanding of PSD in an itemized fashion:

(1) Consistent with the Diagnostic and Statistical Manual of Mental Disorders, Fifth Edition (DSM5) introducing 'spectrum' terminology, we proposed PSD combining all DSM5 psychotic disorders (schizophrenia, schizoaffective disorder, brief psychotic disorder, and so on) to end the illogical emphasis on schizophrenia. Thus, in contrast to DSM5 retaining the distinction between schizophrenia and other psychotic disorders, the concept of PSD takes one step forward towards a true spectrum approach, analogous to autism spectrum in DSM5. We also argue that a complete transition to spectrum approach necessitates renaming to liberate our minds.

(2) We never claimed that schizophrenia is not a mental disorder but argued that schizophrenia is not a natural disease entity, and therefore attempts to reverse-engineer schizophrenia are destined to fail. This concern was raised by the National Institute of Mental Health and led to the founding of the Research Domain Criteria project (Cuthbert \& Insel, 2010).

(3) Evidence indicates substantial neurobiological overlap between bipolar disorder and PSD defying Kraepelin's dichotomy. DSM5 appraised these findings, separated bipolar disorders from depressive disorders, and placed it between the chapters of depressive disorders and schizophrenia spectrum and other psychotic disorders. Given accumulating data, we speculated that bipolar disorder would likely find a place within the framework of PSD in the future. At this stage, we, however, await confirmatory data. We also pointed out the need for transdiagnostic research to embrace heterogeneity inherent to mental disorders, such as the BipolarSchizophrenia Network on Intermediate Phenotypes study (Pearlson et al. 2016).

(4) We never suggested extending the boundaries of PSD to include psychotic experiences and beyond in clinical practice. On the contrary, we questioned the validity of the ultra-high-risk-cum-transition paradigm attributing special value to positive psychotic experiences rather than embracing the full range of person-specific psychopathology in identifying at-risk population - in other words, the 'pre-schizophrenia' group (van Os \& Guloksuz, 2017). However, we do conclude that psychotic experiences, similar to any other subtle expression of psychopathology domains (e.g. autism, anxiety, depression, and cognition), provide an invaluable source of information to leverage our efforts in gaining insight into early psychopathology and mastering the disease phenotype in the research setting, as evidenced by abundant research.

With this opening, we wish the American Psychiatric Association to reconsider their earlier decision to relegate dimensions to the appendix and integrate dimensional assessment of eight psychopathology domains into the main text in the upcoming revision, DSM5.1, particularly in the chapter on psychotic disorders. Even this subtle revision would clear the path to test the utility of the multidimensional spectrum approach in psychosis.

\section{Acknowledgement}

SG and JvO would like to acknowledge the European Community's Seventh Framework Program under grant agreement No. HEALTH-F2-2009-241909 (Project EU-GEI).

\section{Declaration of Interest}

None.

\section{References}

Cuthbert BN, Insel TR (2010). Toward new approaches to psychotic disorders: the NIMH Research Domain Criteria project. Schizophrenia Bulletin 36, 1061-1062.

Guloksuz S, van Os J (2017). The slow death of the concept of schizophrenia and the painful birth of the psychosis spectrum. Psychological Medicine, July 10, 1-16. doi: 10.1017/ S0033291717001775. [Epub ahead of print]. 
Pearlson GD, Clementz BA, Sweeney JA, Keshavan MS, Tamminga CA (2016). Does biology transcend the symptom-based boundaries of psychosis? Psychiatric Clinics of North America 39, 165-174.

van Os J, Guloksuz S (2017). A critique of the 'ultra-high risk' and 'transition' paradigm. World Psychiatry 16, 200-206.

S. GULOKSUZ ${ }^{1,2}$ AND J. VAN Os ${ }^{1,3,4, *}$

${ }^{1}$ Department of Psychiatry and Psychology, Maastricht University Medical Centre, Maastricht, The Netherlands

${ }^{2}$ Department of Psychiatry, Yale University School of Medicine,
New Haven, CT, USA

${ }^{3}$ Department of Psychiatry, Brain Centre Rudolf Magnus, University Medical Centre Utrecht, Utrecht, The Netherlands

${ }^{4}$ Department of Psychosis Studies, King's College London, King's

Health Partners, Institute of Psychiatry, London, UK

*Address for correspondence: J. van Os, Department of Psychiatry, University Medical Centre Utrecht, PO BOX 85500, 3508 GA Utrecht, The Netherlands.

(Email: vanosj@gmail.com) 